\title{
THz parallel plate photonic waveguides
}

\author{
Adam Bingham, Yuguang Zhao, and D. Grischkowsky ${ }^{\text {a) }}$ \\ School of Electrical and Computer Engineering, Oklahoma State University, Stillwater, Oklahoma 74078
}

(Received 24 March 2005; accepted 3 June 2005; published online 26 July 2005)

\begin{abstract}
We have observed narrowband transmission or rejection in the frequency spectra of $\mathrm{THz}$ pulses transmitted through air-spaced parallel plate photonic waveguides. These waveguides have one of the metal plates covered by a silicon plate with a metallic photonic band gap (PBG) surface precisely fabricated by lithographic techniques. We use two different PBG surface types: an array of metallic cylindrical pillars, and an array of metallic cylindrical holes. With the inversion of the PBG structures from cylinders to holes, the output spectra changes from narrow bandpass to narrow band-reject filtering. These photonic waveguides show extremely sharp spectral responses in regions as large as $1 \mathrm{THz}$, with stop bands or transmission bands having contrasts of as much as $90 \mathrm{~dB}$.

(C) 2005 American Institute of Physics. [DOI: 10.1063/1.1997273]
\end{abstract}

Over the past few years the metal parallel plate waveguide (PPWG) has received much attention for its use at $\mathrm{THz}$ frequencies, where it offers transverse electromagnetic (TEM) mode propagation with no modal dispersion, loss determined by the conductivity of the metal plates, and consequent very low group velocity dispersion. ${ }^{1,2}$ With its excellent coupling to free space $\mathrm{THz}$ radiation, and ease of fabrication, the PPWG is the ideal structure for the undistorted guiding of sub-ps $\mathrm{THz}$ pulses. Recently other guided wave components have been integrated into the PPWG for further control over pulse propagation, e.g., metallic mirrors, ${ }^{3}$ transmitters, ${ }^{4}$ and dielectric lenses. ${ }^{5}$ The next technical challenge involves frequency filtering inside the waveguide.

While many groups have demonstrated various filters for $\mathrm{THz}$ applications, most of the recent work has focused on $\mathrm{THz}$ photonic band gap (PBG) structures. A subset of the $\mathrm{THz}$ PBG research has included the integration of these structures into waveguides resulting in a plastic photonic fiber, ${ }^{6}$ and a dielectric waveguide grating. ${ }^{7}$ More recently there has been the first demonstration of a dielectric PBG structure filled $\mathrm{PPWG},{ }^{8}$ giving $\mathrm{THz}$ frequency filtering inside the PPWG.

Due to the lack of spatial dependence of the TEM mode in the direction perpendicular to the plates of the PPWG, three-dimensional PBG cylinder structures can be replicated in the two-dimensional (2D) geometry of the PPWG. ${ }^{5}$ The initial conceptual plan for the experiment presented here was to incorporate such 2D-PBG cylinder structures into the metal PPWG to control the frequency dependent transmission. This goal was to be achieved with high-precision 2DPBG cylinder structures, fabricated by our new cleanroom based lithographic technique. Initially, we used a 2D-PBG structure of metal cylinders to fill the space between the two metal plates of the waveguide. However, the transmission through this structure was negligible. In order to increase the transmission, we broke the 2D-PBG symmetry by increasing the space between the waveguide plates to let the tops of the 70- $\mu \mathrm{m}$-long metal cylinders (pillars) form the surface of a $100 \mu \mathrm{m}$ air gap to the second plate. For this theoretically more complex asymmetric system we observed strong PBG transmission effects. Here, we describe this new class of air-

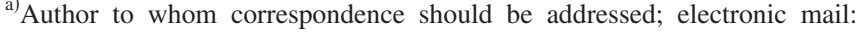
grischd@ceat.okstate.edu spaced parallel plate photonic waveguides, where a metallic PBG surface replaces one of the plates of the PPWG. The two complementary PBG surfaces shown in Fig. 1 allow controllable frequency filtering based on the dimensions, array design, and periodicity of the sample. The resulting $\mathrm{THz}$ photonic waveguides give high contrast frequency filtering, with the added benefits of air propagation and standard lithographic fabrication.

Although the use of corrugations or surface modulation on the plates of a PPWG has been theoretically studied, ${ }^{9-11}$

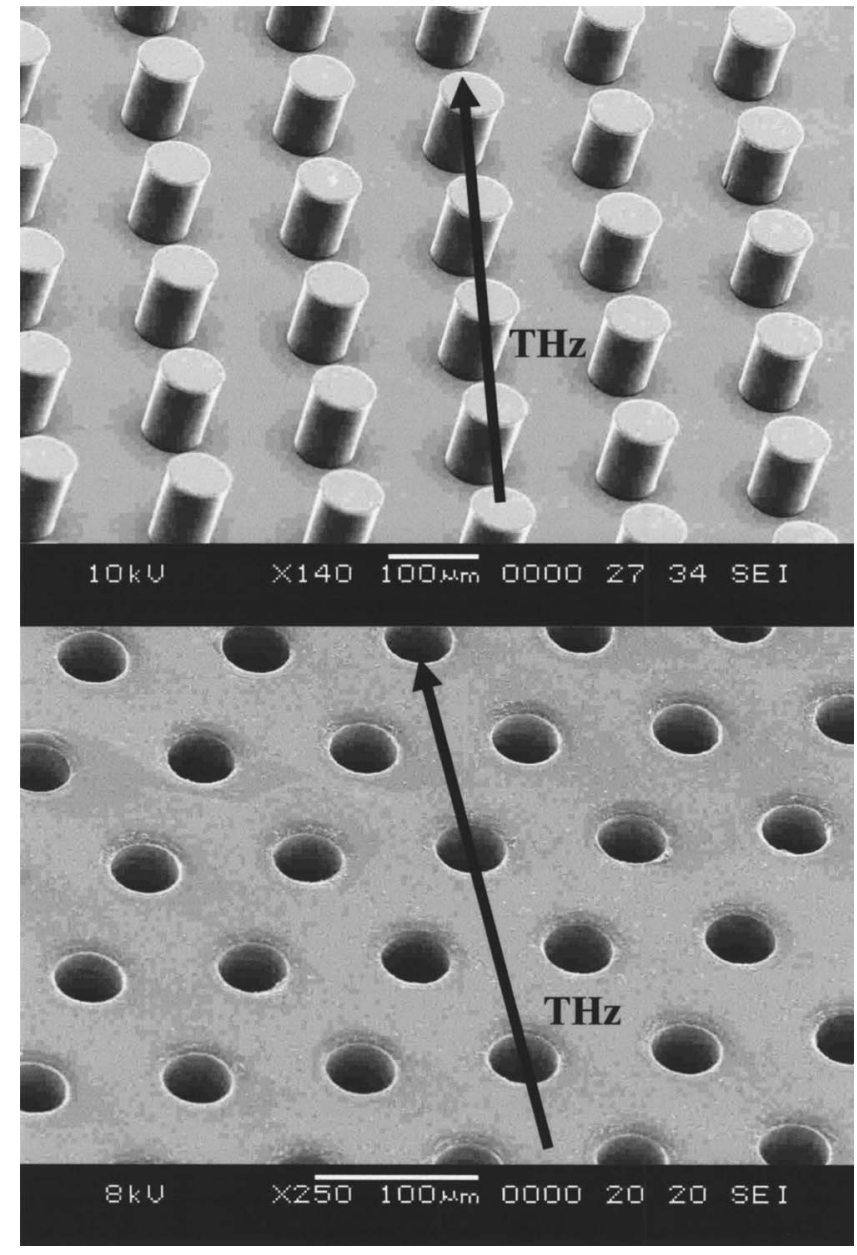

FIG. 1. Upper SEM image is the type I PBG surface. Lower SEM image is the type II PBG surface. 
the use of PBG surfaces ${ }^{12}$ to control the frequency content of guided wave electromagnetic radiation appears to be more versatile and effective. In one of the first demonstrations, a PBG surface was used to suppress microwave surface waves in a desired frequency range. ${ }^{12}$ Following this concept, other experiments integrated PBG surfaces into microwave waveguides. ${ }^{13,14}$ Applications have included suppressing parallel plate noise in printed circuit boards, ${ }^{13}$ creating a rectangular microwave waveguide capable of propagating radiation in the TEM mode, ${ }^{14}$ and the integration of a PBG surface into a PPWG to create an asymmetric parallel plate Luneburg lens. ${ }^{15}$ The most comparable work to our THz parallel plate photonic waveguides is the $2 \mathrm{D}$ Bragg resonator, as used in a free electron maser resonator. ${ }^{16,17}$ For the $2 \mathrm{D}$ Bragg resonator, diamond shaped corrugations were machined into the surfaces of a parallel plate waveguide, for sharp frequency resonances. While all of these microwave structures share similarities with the $\mathrm{THz}$ parallel plate photonic waveguide, typically the depth of the corrugations on the microwave PBG surface were much smaller than a wavelength, allowing for a simplified theoretical understanding in terms of capacitive and inductive elements. For our $\mathrm{THz}$ demonstrations, the corrugation depth is on the order of a wavelength, creating effective frequency response, but together with a more difficult theoretical problem.

In order to make the high quality metallic $2 \mathrm{D}$ photonic surfaces shown in Fig. 1, we employ photolithography and metal deposition techniques, as opposed to the traditional etching methods, which can cause undercutting and rough surfaces. By using lithography, the cylinders or holes are uniform without undercutting. Due to the use of photoresist, the sidewalls are smoother than for etched holes. As the initial novel processing step, SU-8 2025 negative photoresist from MicroChem Inc. is spun onto a 3 in., 400- $\mu$ m-thick silicon wafer to form an unusually thick $70 \mu \mathrm{m}$ film. After standard photolithography with custom designed masks followed by high temperature baking, the PBG structured film becomes permanent. The $\mathrm{Si}$ wafer is then diced into oriented 25.4-mm-square plates to fit the plates of the waveguide. The final process is metallization of the PBG surface. Approximately $300 \mathrm{~nm}$ of gold is deposited by a sputter coater, which has excellent step coverage.

Different masks with submicron-dimensional precision were used to fabricate a variety of diameters of both cylinders and holes in either square or hexagonal arrays. Figure 1 shows scanning electron microscope (SEM) images of the two PBG surfaces studied in this letter. The upper image shows the type I surface consisting of 70- $\mu \mathrm{m}$-tall cylindrical pillars in a planar square array. Although the array was fabricated using a lithographic mask that has precise $60-\mu \mathrm{m}$-diameter holes with a $160 \mu \mathrm{m}$ array period, the diameter of the cylinders increased to approximately $70 \mu \mathrm{m}$ during the development process. The lower SEM image shows the type II surface consisting of 70- $\mu \mathrm{m}$-deep holes in a hexagonal array. For this pattern, the mask had an array period of $100 \mu \mathrm{m}$, and a hole diameter of $60 \mu \mathrm{m}$. During the development process, the diameter of the holes decreased to approximately $40 \mu \mathrm{m}$.

The resulting parallel plate photonic waveguides were characterized by $\mathrm{THz}$ time-domain spectroscopy (THz-TDS). ${ }^{1}$ The $\mathrm{THz}$ reference pulses were transmitted through a 25.4-mm-square Cu PPWG with $100 \mu \mathrm{m}$ plate separation. Two plano-cylindrical silicon lenses are used to Downloaded 01 Aug 2005 to 139.78.79.26. Redistribution subjec

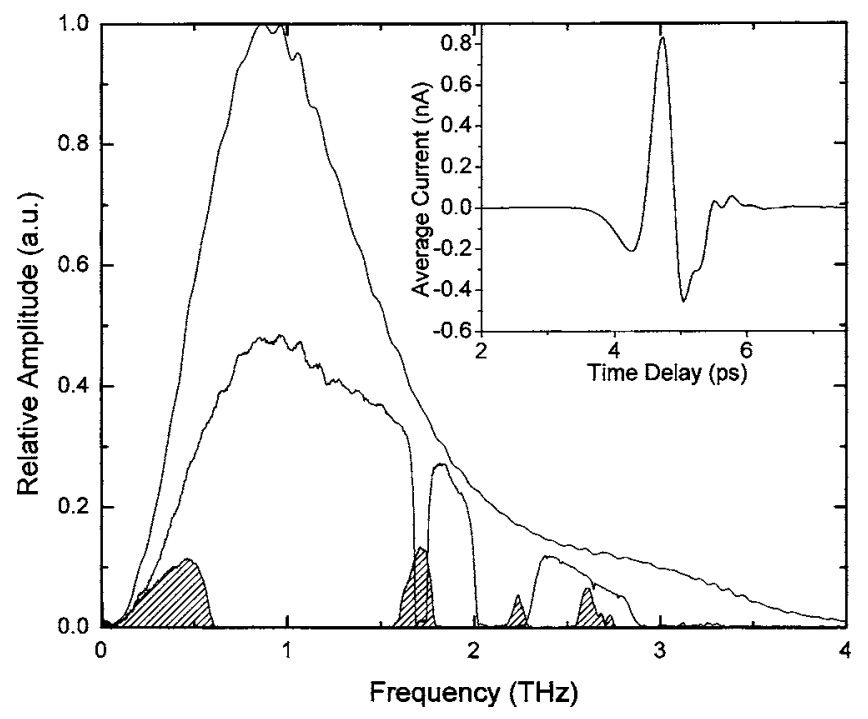

FIG. 2. Normalized amplitude spectra from the reference (upper curve) and sample pulses. The middle spectrum is from the type II photonic waveguide and the shaded spectrum is from the type I photonic waveguide. Inset: Reference $\mathrm{THz}$ pulse.

couple the radiation into and out of the waveguide, placed at the confocal beam waist of the THz-TDS system. For the photonic waveguides, $\mathrm{Al}$ waveguides are used, and a Si plate with a metallic PBG surface is placed over and in contact with one Al plate; $100 \mu \mathrm{m}$ edge spacers are placed between the other Al plate and the metallic PBG surface. The propagation directions relative to the PBG surfaces are indicated in Fig. 1. The incoming THz pulse is polarized perpendicular to the plate surfaces.

Our measured THz reference pulse is shown as the inset in Fig. 2, which also shows the normalized amplitude spectra of the reference pulse and the two experimental pulses, demonstrating the powerful filtering capability of the two photonic waveguides. The complete measurements are shown in Figs. 3 and 4. Figure 3(a) shows the THz pulse transmitted through the type I photonic waveguide; four output pulses were averaged to improve the signal to noise ratio. The measured pulse extends to $130 \mathrm{ps}$, but has been truncated for better illustration. Figure 3(b) shows the amplitude spectrum of the entire pulse, and Fig. 3(c) shows corresponding power transmission in dB. Figure 4(a) shows the average of four output pulses from the type II photonic waveguide. The THz output pulse had a shorter ringing structure of only 100 ps, and Fig. 4(a) shows a truncated version of the pulse. Figure 4(b) shows the amplitude spectrum of the entire pulse, and Fig. 4(c) shows the power transmission $T(\omega)$ in dB. All of the output data are normalized to the reference pulse. The power transmission in $\mathrm{dB}, T(\omega)=20 \log |S(\omega) / R(\omega)|$, where $S(\omega)$ is the sample spectrum and $R(\omega)$ is the reference spectrum shown in Fig. 2.

Figure 3(b) shows a particularly wide band gap, from 0.6 to $1.55 \mathrm{THz}$, with a transmission contrast of $90 \mathrm{~dB}$. Well resolved $90 \mathrm{~dB}$ transmission peaks are also observed, illustrating the efficacy and potential for applications of the type I waveguide. In contrast to these observations the highly transmissive narrow band-reject capability of the type II waveguide is shown in Figs. 4(b) and 4(c). The observed band rejection has a contrast of approximately $65 \mathrm{~dB}$. However, the very sharp Bragg rejection resonance at $1.692 \mathrm{THz}$ is reproducible, has an instrument-limited linewidth of 10
to AIP license or copyright, see http://apl.aip.org/apl/copyright.jsp 

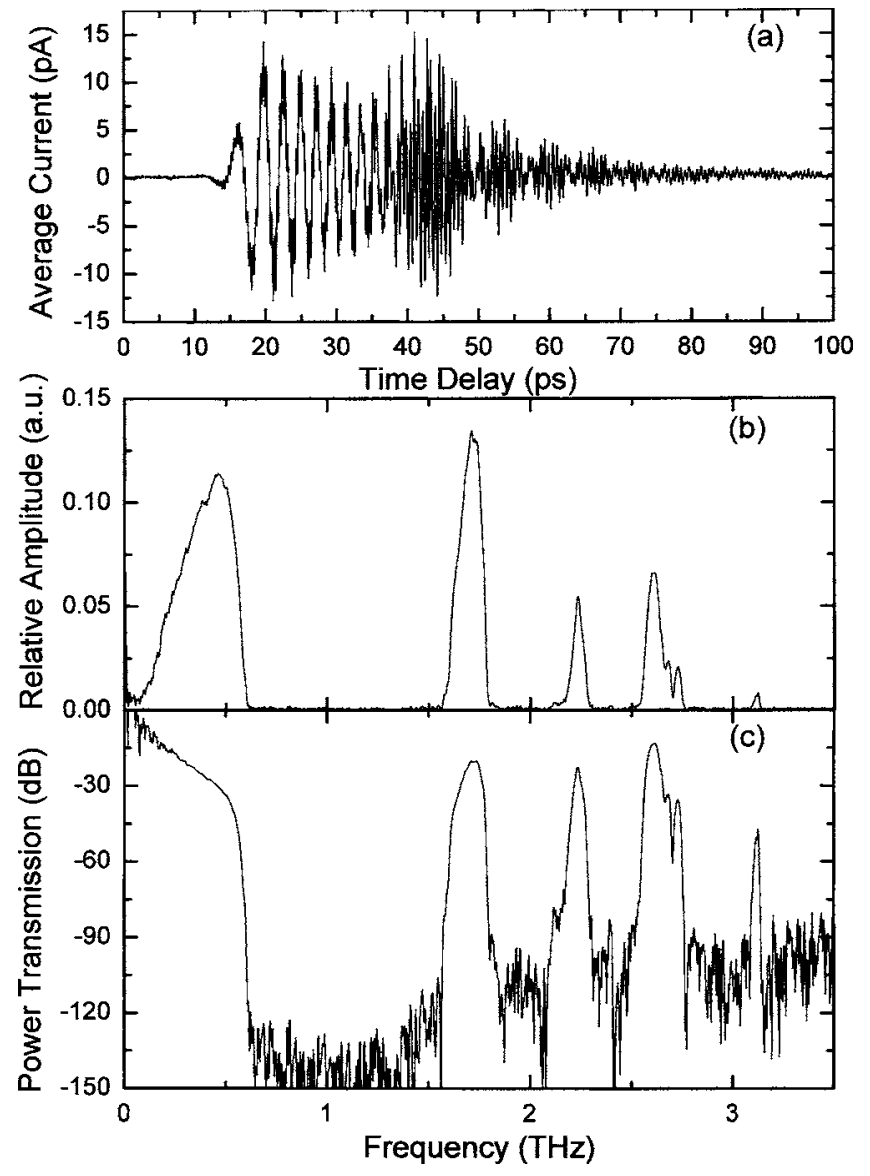

FIG. 3. (a) Output THz pulse from type I photonic waveguide. (b) Amplitude spectrum of output pulse normalized to reference pulse. (c) Power transmission spectrum in $\mathrm{dB}$.

$\mathrm{GHz}$, and a rejection contrast of $100 \mathrm{~dB}$. This resonance corresponds to in-phase reflections from the adjacent hole lines perpendicular to the $\mathrm{THz}$ propagation direction shown in the lower part of Fig. 1. The $177.3 \mu \mathrm{m}$ wavelength corresponding to $1.692 \mathrm{THz}$ is compared with $173.2 \mu \mathrm{m}$, twice the $86.6 \mu \mathrm{m}$ separation between the adjacent hole lines, thereby indicating a reduced phase velocity of $c / 1.024$ of the type II photonic waveguide. Also, note the high transmission with less than $15 \mathrm{~dB}$ loss over the transmitted portion of the spectrum out to $3 \mathrm{THz}$. While both photonic waveguides are dispersive, the low frequency $(<1.5 \mathrm{THz})$ main peak of the transmitted pulse from the type II waveguide arrives with little delay. The higher frequency components follow in the ringing structure. Essentially, the type II waveguide acts as an ordinary PPWG for low frequencies, with filtering at the high frequencies. In contrast, the type I waveguide transmitted pulse shows an 11.9 ps time delay relative to the type II waveguide, as well as a more dispersive and much longer ringing structure.

In conclusion, we have demonstrated a new class of airspaced, metal parallel plate photonic waveguides for either narrowband-pass or narrowband-reject high contrast frequency filtering. Due to the high lithographic precision of the investigated type I and II PBG surfaces, the consequent sharp, high contrast filtering observations present a wellcharacterized challenge for the development of a suitable PBG theory (likely to be numerical) for their detailed explanation. Such a theory, coupled with the flexibility and submicron precision of lithographic mask design, would enable
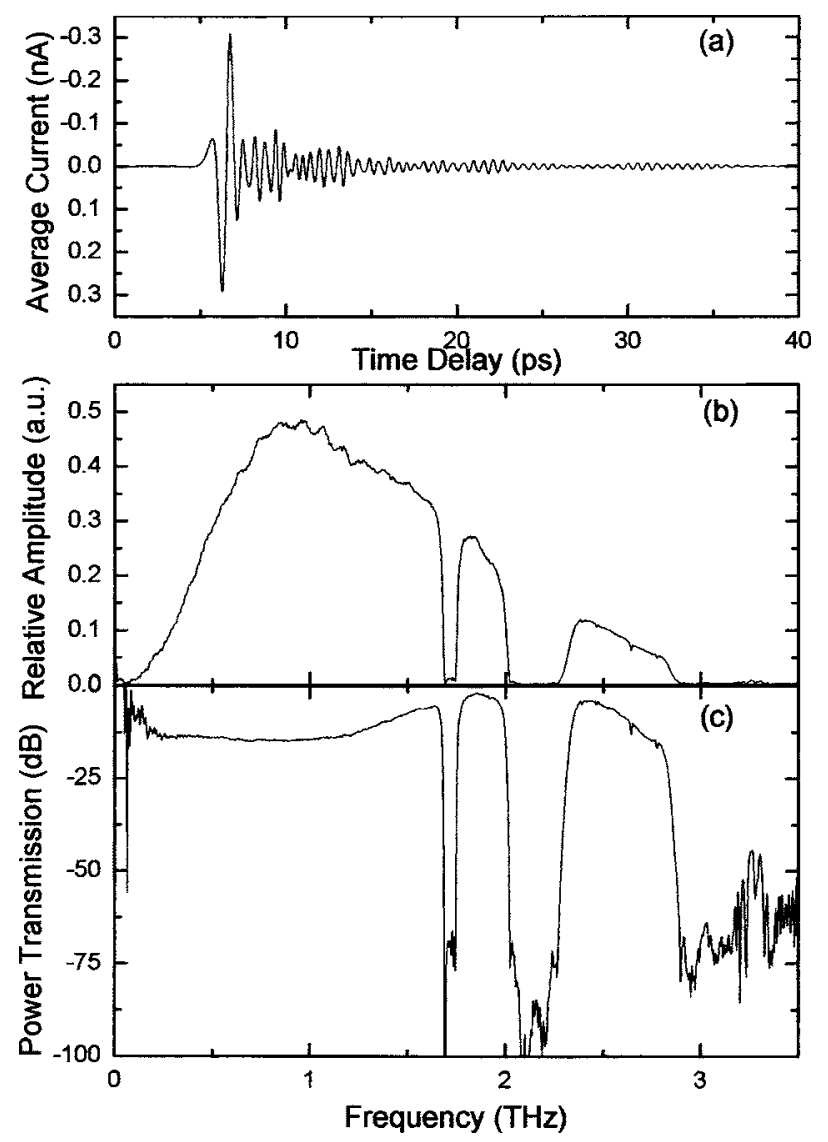

FIG. 4. (a) Output THz pulse from type II photonic waveguide (b) Amplitude spectrum of output pulse normalized to reference pulse. (c) Power transmission spectrum in $\mathrm{dB}$.

the fabrication of parallel plate photonic waveguides with specified characteristics.

The author's acknowledge informative discussions with Dr. Jiangquan Zhang. This work was partially supported by the National Science Foundation and the U.S. Department of Energy.

${ }^{1}$ R. Mendis and D. Grischkowsky, Opt. Lett. 26, 846 (2001).

${ }^{2}$ R. Mendis and D. Grischkowsky, IEEE Microw. Wirel. Compon. Lett. 11, 444 (2001).

${ }^{3}$ S. Coleman and D. Grischkowsky, Appl. Phys. Lett. 833656 (2003).

${ }^{4}$ S. Coleman and D. Grischkowsky, Appl. Phys. Lett. 84, 654 (2004).

${ }^{5}$ J. Dai, S. Coleman, and D. Grischkowsky, Appl. Phys. Lett. 85, 884 (2004).

${ }^{6}$ H. Han, H. Park, M. Cho, and J. Kim, Appl. Phys. Lett. 80, 2634 (2002). ${ }^{7}$ J. Roux, F. Aquisapace, F. Garet, L. Duvillaret, and J. L. Coutaz, Appl. Opt. 41, 6507 (2002).

${ }^{8}$ Z. Jian, J. Pearce, and D. Mittleman, Opt. Lett. 29, 2067 (2004).

${ }^{9}$ A. Nayfeh and O. Asfar, J. Appl. Phys. 45, 4797 (1974).

${ }^{10}$ O. Asfar and A. Nayfeh, IEEE Trans. Microwave Theory Tech. MTT-28, 1187 (1980).

${ }^{11}$ S. Sandstrom, J. Acoust. Soc. Am. 79, 1293, (1986).

${ }^{12}$ D. Sievenpiper, L. Zhang, R. Broas, N. Alexopolous, and E. Yablonovitch, IEEE Trans. Microwave Theory Tech. 47, 2059 (1999).

${ }^{13}$ R. Abharis and G. Eleftheriades, IEEE Trans. Microwave Theory Tech. 51, 1629 (2003).

${ }^{14}$ F. Yang, K. Ma, Y. Quan, and T. Itoh, IEEE Trans. Microwave Theory Tech. 47, 2092 (1999).

${ }^{15}$ Y. Park, A. Herschlein, and W. Wiesbeck, IEEE Trans. Microwave Theory Tech. 47, 2092 (2002).

${ }^{16}$ N. Ginzburg, N. Peskov, and A. Sergeev, Opt. Commun. 96, 254 (1993).

${ }^{17}$ N. Peskov, N. Ginzburg, G. Denisov, A. Sergeev, A. Arzhannikov, S. Sinitsky, P. Kalinin and V. D. Stepanov, Opt. Commun. 187, 311 (2001). 